\title{
Photoluminescence Microscopy of Single Impurities and Defects in PV Materials
}

\author{
Angelo Mascarenhas and Brian Fluegel \\ National Renewable Energy Laboratory, Golden, Colorado, 80401, USA
}

Using optical spectroscopy with diffraction limited spatial resolution, it is possibile to study the luminescence from single impurity centers in a semiconductor. The dilute nitride alloy $\mathrm{GaAs}_{1-\mathrm{x}} \mathrm{N}_{\mathrm{x}}$ is of interest as a $1 \mathrm{eV}$ absorber photovoltaic material. The electronic bandstructure of this alloy emerges from the interaction between nitrogen impurities and the GaAs host. By selectively studying individual impurity centers that are formed by two neighboring nitrogen atoms in GaAs as shown in Fig. 1, it possible to unveil their selection rules, identify their particular impurity configuration, map their spatial distribution, and demonstrate the presence of a diversity of local environments. Circumventing the limitation imposed by ensemble averaging and the ability to discriminate the individual electronic responses from discrete emitters provides an unprecedented perspective on the nanoscience of impurities.

The ternary semiconductor alloy $\mathrm{GaInP}_{2}$ plays a very prominent role in High-Efficiency Multijunction Solar Cells as well as in Solid-State Lighting. The size-mismatch induced strain between the binary components leads to interesting phenomena such as spontaneous ordering. Ordering has been shown to result in dramatic changes to the electronic and optical properties of this alloy and over the past two decades there have been extensive studies on the intrinsic and extrinsic changes that result from this phenomenon. In addition to bandgap lowering, spontaneous ordering is accompanied by defect-induced changes in the PL spectrum which are poorly understood. A broad band low energy band (LEB) is uniformly present, and on a microscopic scale, extremely sharp emission lines (LEL) are seen in some samples. Attempts have been made to explain the LEL as transitions from regions where stacking faults induce a quantum-confined heterostructures, however these fail to explain either the total dependence on sample growth, or the complete omnipresence of the LEL in samples where they do appear. We have now developed a novel photoluminescence technique to probe the microstructure of spontaneously ordered $\mathrm{GaInP}_{2}$. The photoluminescence (PL) mapping used here is a direct-imaging technique in which the entire 2D sample surface is imaged onto an electron-multiplying-CCD camera. Spectral resolution is obtained by an LCDtunable bandpass filter that dynamically selects the PL band of interest.. Fig. 2a reveals new spatial details that are now uncovered by examining a large area in spectral regions where no macroscopic signal is found. The bright points seen in Fig. 2a, and whose spectrum is labeled VLEB in Fig. $2 b$ are found to occur in the same samples that show the LEL. This correlation, and their lower energy, indicate a possible compound defect, whose density is much lower than their parent defects which cause the LEL. Further study of these new features may illuminate the nature of the low-energy emission in ordered GaInP. 
(a)

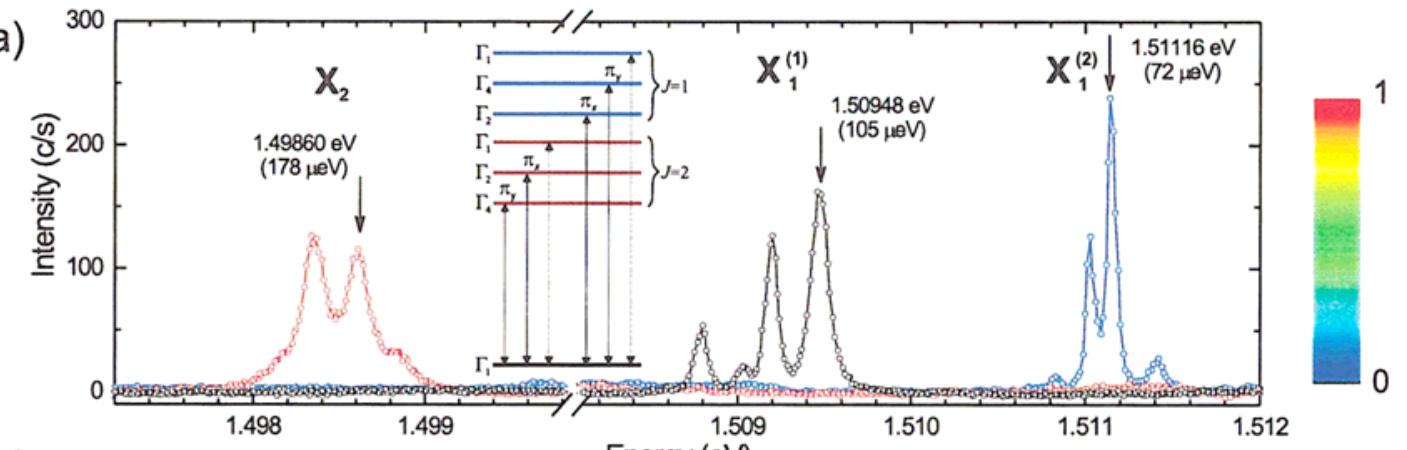

(b)
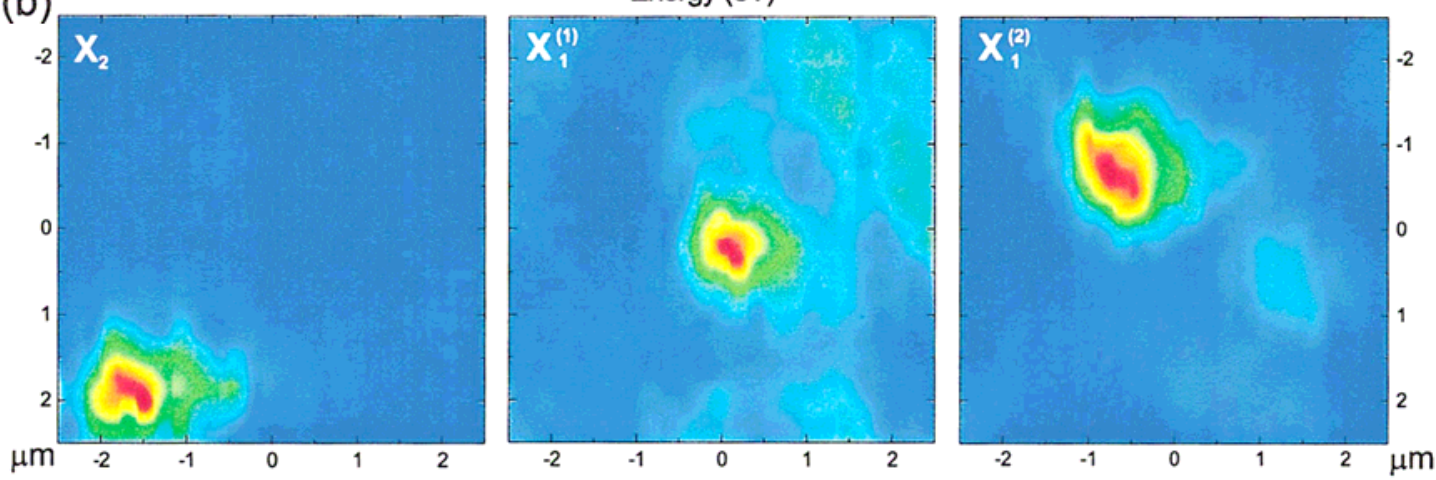

Fig.1 Photoluminescence spectra obtained from three different regions of a $25 \mu \mathrm{m}$ spatial map in a $\mathrm{GaAs}_{1-\mathrm{x}} \mathrm{N}_{\mathrm{x}}$ sample. $X_{1}^{(1)}, X_{1}^{(2)}$, and $X_{2}$ correspond to three distinct nitrogen pairs. Inset: linearly polarized optical transitions for $C_{2 v}$ symmetry. The photon wave vector is along $z$, the $C_{2}$ axis, and the pair is oriented along $x$. (b) Photoluminescence intensity maps corresponding to pairs $X_{1}^{(1)}, X_{1}^{(2)}$, and $X_{2}$. The intensity is plotted for the luminescence peaks shown by arrows on the spectra in (a).

(a)
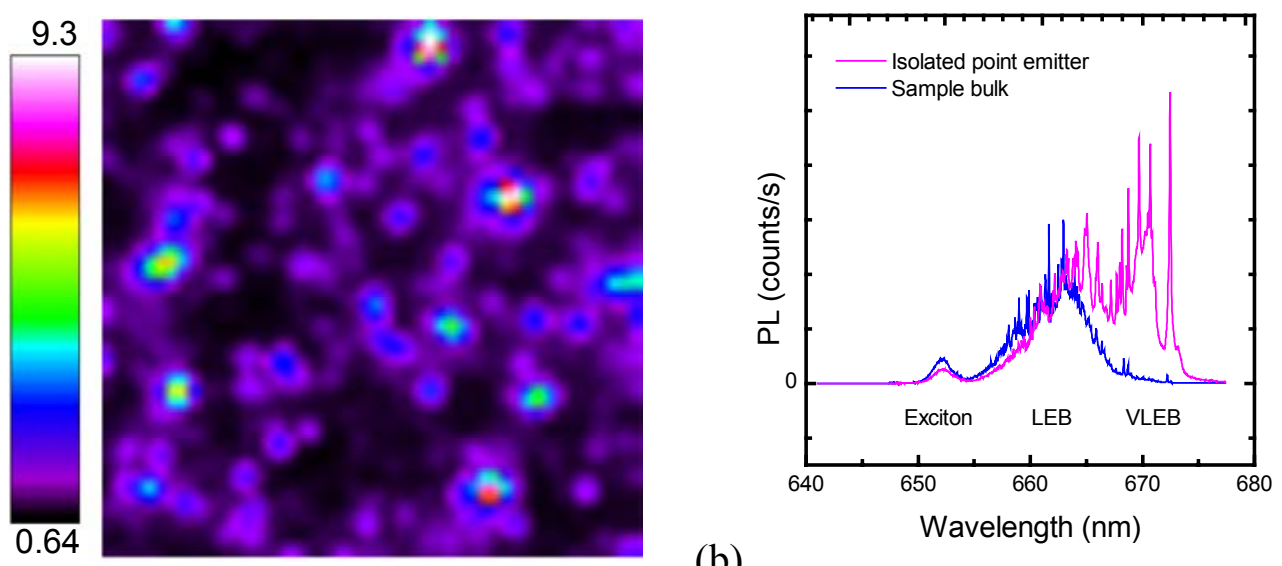

Fig. 2. (a) A PL map of the new very low-energy spectral region of $7 \mathrm{~K}$ ordered GaInP. The spatial extent is 20 microns. Within in this 5-nm wide band centered at $675 \mathrm{~nm}$, numerous isolated submicron emission centers are seen. (b) Spectra of a single isolated bright spot from Fig. 2a, and a sample point from the bulk (dark regions in Fig. 2a). The bandedge PL, low-energy band, and accompanying sharp lines are largely unchanged in the bright spots, with the main difference occurring in the addition of a new spectral band labeled VLEB. 